\title{
Definición de suicidio y de los pensamientos y conductas relacionadas con el mismo: una revisión
}

\author{
Definition of suicide and of the thoughts \\ and behaviors related to it: $A$ review
}

\author{
José Nahum Rangel Villafaña y Samuel Jurado Cárdenas \\ Universidad Nacional Autónoma de México ${ }^{1}$
}

Autor para correspondencia: José Nahum Rangel V.,joserangel_2@hotmail.com.

\section{RESUMEN}

\begin{abstract}
Se calcula que en un año se cometen más de 800,000 suicidios en el mundo, uno cada 40 segundos. En México, se reportaron 6,559 suicidios en el año 2017. La tasa de suicidios ha aumentado, lo que representa un fenómeno con un alto impacto social. Para su prevención, es necesario abordar los pensamientos y conductas relacionadas al suicidio mediante estrategias basadas en la evidencia. Para ello, las definiciones conceptuales y operacionales desempeñan un papel crucial en cuanto que influyen directamente en la validez interna de los estudios efectuados sobre esta área. Por esta razón, el objetivo del presente texto fue analizar una parte de la literatura internacional acerca de la definición de suicidio, así como las propuestas para definir las conductas y pensamientos relacionados con tal fenómeno, considerándose las tres más utilizadas. Así, se enlistan los términos que ya son inaceptables y se hacen sugerencias para los profesionales de la salud en México.
\end{abstract}

Palabras clave: Suicidio; Ideación suicida; Intento de suicidio; Nomenclatura; Clasificación.

\begin{abstract}
Every year more than 800,000 suicides occur globally, roughly one every 40 seconds. In Mexico, 6,559 suicides were reported in 2017, which showed an increase in the suicide rate, implying a high social-impact phenomenon. Researchers and professionals need to address thoughts and behaviors related to suicide with evidence-based strategies to prevent these trends. Reliable conceptual and operational definitions perform a crucial role toward this purpose as they directly relate to the internal validity of studies in the area. Thus, the objective of this paper was to partially analyze the international research literature on the definition of suicide and various proposals to define the thoughts and behaviors related to this phenomenon. Results point to the three most used related thoughts, which lead to suggestions for health professionals in Mexico.
\end{abstract}

Key words: Suicide; Suicidal ideation; Suicide attempt; Nomenclature; Classification.

\footnotetext{
${ }^{1}$ Facultad de Psicología, Av. Universidad 3004, Col. Copilco-Universidad, Coyoacán, 04510, Ciudad de México, México, tels. (55)55-20-85-83-73 y(55)55-18-50-15-84, correos: joserangel_2@hotmail.com y jurado@unam.mx.
} 
Recibido: 21/08//2020

Aceptado: 01/12/2020

$\mathrm{E}$ 1 suicidio figura como un problema que tiene un alto impacto social. La Organización Mundial de la Salud (WHO) (2012) contabilizó alrededor de 800 mil suicidios cada año en el mundo, es decir, un suicido cada 40 segundos. Además, se calcula que se hacen veinte intentos de suicidio por cada suicidio. En México, el número de defunciones por suicidio en 2017 fue de 6,559 , lo que representa $1 \%$ del total de muertes en ese año, de las cuales 5,323 fueron de hombres y 1,233 de mujeres. Entre las personas de 15 a 39 años se comete $62 \%$ de esos fallecimientos, esto es, 4,088 (Instituto Nacional de Estadística, Geografía e Informática [INEGI], 2019).

Otro factor de relevancia para México es el aumento en las defunciones por suicidio (Borges, Orozco, Benjet y Medina-Mora, 2012). De hecho, la cifra de 1.13 por cada 100,000 habitantes, observada en 2007, llegó a 5.0 en 2015 (OMS, 2018a).

Con base en lo anterior, es esencial trabajar en la prevención de este fenómeno, particularmente en el tratamiento de los pensamientos y las conductas relacionadas con el suicidio. Para ello, es indispensable implementar intervenciones basadas en la evidencia (Glasziou, Del Mar y Salisbury, 2004; Rubin y Bellamy, 2012) para lo cual la investigación en el área del suicidio es imperativa (O’Connor y Nock, 2014).

Silverman y De Leo (2016) concluyeron que es indispensable contar con una nomenclatura internacional para referirse a los pensamientos $\mathrm{y}$ conductas vinculadas al suicidio. Un instrumento así facilitaría la comunicación entre los profesionales de la salud, las definiciones operacionales en el área y la investigación, eliminaría el uso de términos confusos o peyorativos y contribuiría a reducir el estigma relacionado con el fenómeno.

Específicamente en cuanto a la atención de las personas, Silverman (2006) enlistó algunas ventajas que proporcionaría una nomenclatura internacional estandarizada: mejoraría la claridad, precisión y consistencia al evaluar y atender a las personas; mejoraría la comunicación entre los profesionales de la salud; facilitaría la toma de decisiones para la evaluación y el tratamiento basado en la evidencia; eliminaría la terminología que pudiera ser peyorativa, y mejoraría la comunicación de la díada profesional de la salud y persona en atención.

Lo anterior destaca la relevancia del presente escrito, pues para conducir cualquier tipo de investigación con control experimental para alcanzar un mayor nivel de validez interna es necesario contar con una definición conceptual y operacional precisa (Kerlinger y Lee, 2002).

Por lo hasta aquí dicho, el objetivo del presente trabajo fue analizar parte de la literatura internacional acerca de la definición conceptual del suicidio, así como las propuestas para definir los pensamientos y conductas relacionados con este fenómeno.

\section{Definición de suicidio}

Es común que el profesional de la salud recurra a la Clasificación Internacional de Enfermedades (CIE) (OMS, 1990) para definir o categorizar la condición que está tratando. Efectivamente, la CIE en su décima edición, dispone de un apartado al respecto; sin embargo, la citada definición no se revisa a profundidad en este texto en virtud de que la clasificación que se expone sobre las lesiones autoinfligidas intencionalmente solamente está en función del método utilizado para la lesión (OMS, 1990; OMS, 2018b).

Se puede considerar la publicación de Durkheim (1897) como el inicio de la investigación cuantitativa en el campo del suicidio. En su propuesta sociológica sobre el suicidio, definió al fenómeno como "todo caso de muerte que resulte directa o indirectamente de un acto positivo o negativo, ejecutado por la propia víctima, a sabiendas de que habría de producir este resultado" (p. 14).

Con el paso de los años, se han postulado diversas definiciones sobre el suicidio. A continuación se enlistan algunas de las más relevantes: Shneidman (1985), por ejemplo, considera al suicidio como un acto consciente de aniquilación autoinducida, mejor entendida como un malestar multidimensional en las necesidades de un indi- 
viduo que representa un problema para el cual el suicidio se percibe como la mejor solución. Por otro lado, la Organización Mundial de la Salud y la Organización Panamericana de la Salud (WHOPHO) (1998) definieron el suicidio como el acto de quitarse la vida deliberadamente, iniciado y realizado por la persona, en pleno conocimiento o con la expectativa de un resultado fatal. De Leo, Burgis, Bertolote, Kerkhof y Bille-Brahe (2004) también lo consideraron un comportamiento con desenlace fatal en el que la persona, sabiendo o esperando ese resultado, inició y condujo la conducta para alcanzar su objetivo.

Una de las conceptualizaciones más actualizadas es la de Crosby, Ortega y Melanson (2011), quienes detallan el suicidio como la muerte resultante del comportamiento autoinfligido perjudicial en el que la persona tenía la intención de morir como resultado de dicha conducta.
No obstante ser propuestas que datan de diferentes años y hechas por distintos autores, se pueden identificar ciertos factores comunes en ellas: la muerte de la persona, una conducta autoinfligida y la intención de morir como resultado de tal comportamiento. En otras palabras, los expertos en el campo del suicidio han logrado un consenso sobre el concepto de suicidio, aunque no en cuanto a los pensamientos y conductas relacionadas con aquel. Por dicha razón, se revisan a continuación las propuestas antes mencionadas.

\section{La nomenclatura de 1996}

O'Carroll et al. (1996) identificaron la intención de morir (presente o ausente) y los resultados de la conducta como factores determinantes para su nomenclatura. En la Tabla 1 se muestra dicha propuesta.

Tabla 1. Nomenclatura para conductas asociadas al suicidio (O'Carroll et al., 1996).

\begin{tabular}{|c|c|c|c|}
\hline & & $\begin{array}{l}\text { Términos para los comportamientos } \\
\text { relacionados con el suicidio }\end{array}$ & Intención de morir \\
\hline \multirow{6}{*}{$\begin{array}{l}\text { Comportamientos } \\
\text { relacionados } \\
\text { con el suicidio }\end{array}$} & \multirow{3}{*}{ Conducta instrumental } & $\begin{array}{l}\text { Conducta instrumental relacionada con el suicidio } \\
\text { con lesiones }\end{array}$ & \multirow{3}{*}{ Ausente } \\
\hline & & $\begin{array}{l}\text { Conducta instrumental relacionada con el suicidio } \\
\text { sin lesiones }\end{array}$ & \\
\hline & & $\begin{array}{l}\text { Conducta instrumental relacionada con el suicidio } \\
\text { con resultado fatal }\end{array}$ & \\
\hline & \multirow{3}{*}{ Actos suicidas } & Intento suicida con lesiones & \multirow{3}{*}{ Presente } \\
\hline & & Intento suicida sin lesiones & \\
\hline & & Suicidio & \\
\hline
\end{tabular}

Los comportamientos relacionados con el suicidio incluyen conductas potencialmente autolesivas para las cuales existe evidencia explícita o implícita de que (a) la persona tuvo la intención de suicidarse, o (b) la persona deseó utilizar la apariencia de su conducta para alcanzar otro objetivo (no hay intención suicida).

La conducta instrumental relacionada con el suicidio se refiere a los comportamientos potencialmente autolesivos para los que existe evidencia explícita o implícita de que: (a) la persona no tenía intención de suicidarse, y (b) la persona deseó utilizar la apariencia de su conducta para alcanzar otro objetivo (pedir ayuda, recibir atención de los demás, etc.).
Por otro lado, los actos suicidas son conductas potencialmente autolesivas para las que existe evidencia explícita o implícita que la persona tuvo la intención de suicidarse. Se considera como suicidio la muerte por lesión, envenenamiento o asfixia, sobre la cual existe evidencia (explícita o implícita) de que la lesión fue autoinfligida y de que la persona fallecida deseaba, en efecto, morir. El intento de suicidio implica un comportamiento potencialmente autolesivo con un resultado no fatal (la persona no murió), sobre el que existe evidencia explícita o implícita de que la persona deseaba terminar con su vida. En el intento suicida puede haber o no lesiones. 
Una limitación de dicha nomenclatura es su falta de precisión respecto a los pensamientos relacionados con el suicido; de hecho, sus autores solo definieron la ideación suicida como cualquier pensamiento en el que se involucre alguno de los comportamientos relacionados con el suicidio. Asimismo, en cuanto a los comportamientos que podrían anteceder a los relacionados con el suicidio, únicamente describieron la amenaza suicida (O’Carroll et al., 1996).

Es importante considerar que dichos autores propusieron una nomenclatura inicial, no concluida, solicitando que su propuesta fuera analizada y criticada para poder mejorarla. Para comprender esta idea, es preciso entender la diferencia entre nomenclatura y clasificación.

Una nomenclatura es un conjunto de términos comúnmente entendidos, definidos lógicamente. Una clasificación, a su vez, implica diversos elementos que hacen posible una mayor precisión; cabe mencionar que una clasificación es exhaustiva, es decir, considera la totalidad de los conceptos y una disposición sistemática de los mismos en categorías y subcategorías ordenadas; tiene validez científica; cuenta con precisión suficiente para la investigación o la práctica clínica, y depende de un conjunto inequívoco de reglas para asignar los conceptos a un solo lugar en la clasificación. En este sentido, la nomenclatura provee el lenguaje para la clasificación, y esta a su vez proporciona la organización de relaciones conceptuales $\left(\mathrm{O}^{\prime} \mathrm{Ca}\right.$ rroll et al., 1996; Posner, Brodsky, Yershova, Buchanan y Mann, 2014).

\section{La nomenclatura de 2007}

Luego de diez años, la propuesta de O'Carroll et al. (1996) se sometió a revisión. En ella, se mantuvo la relevancia de la intención suicida y la letalidad del comportamiento. Asimismo, se descartaron conceptos tales como "gesto suicida", "conducta parasuicida" y "suicidio consumado", sobre todo por la connotación peyorativa que implicaban (Silverman, Berman, Sanddal, O'Carroll y Joiner, 2007a).

Silverman, Berman, Sanddal, O'Carroll y Joiner (2007b) propusieron en su lugar una nomen- clatura con tres categorías: los pensamientos relacionados con el suicidio, las comunicaciones asociadas al suicidio y las conductas asociadas con el suicidio. La ideación relacionada con el suicidio incorpora un evento exclusivamente cognitivo, el cual puede o no involucrar la intención de quitarse la vida. De esta categoría se generan tres términos que se pueden utilizar: a) Ideación relacionada con el suicidio sin intención suicida; $b$ ) Ideación relacionada con el suicidio con intención suicida indeterminada, y c) Ideación relacionada con el suicidio con intención suicida.

Las comunicaciones asociadas al suicidio incorporan dos fenómenos: la amenaza y el plan, ninguno de los cuales entraña una lesión autoinfligida. La amenaza suicida es cualquier acción interpersonal (verbal o no) sin un componente autolesivo directo, la cual puede interpretarse como una sugerencia de que un comportamiento asociado al suicidio podría ocurrir en el futuro próximo. De esta subcategoría se desprenden tres términos que están en función de la intención de quitarse la vida: Amenaza de suicidio I (sin intención), Amenaza de suicidio II (intención indeterminada) y Amenaza de suicidio III (con intención).

El plan suicida, por su parte, involucra una formulación sistemática de un método de acción que tiene el potencial para resultar en una conducta relacionada con el suicidio. Para esta subcategoría también se establecen tres términos diferenciados por la intención suicida: Plan suicida I (sin intención), Plan suicida II (intención indeterminada) y Plan suicida III (con intención).

En la Tabla 2 se muestran conductas relacionadas con el suicidio: autolesión, intento suicida y conducta indeterminada relacionada con el suicidio. Cada una de ellas está generada por la intención de suicidarse y por los resultados del comportamiento (Silverman et al., 2007a).

La revisión efectuada por Silverman et al. (2007a, 2007b) mostró un avance importante en la comprensión del fenómeno debido a que incorporó la conducta indeterminada relacionada con el suicidio, la cual permite agrupar aquellos casos en los que, con base en la información disponible, no es posible establecer la intención suicida. 
Tabla 2. Nomenclatura para las conductas relacionadas con el suicidio (Silverman et al., 2007a).

\begin{tabular}{|c|c|c|c|}
\hline & & $\begin{array}{l}\text { Términos para las conductas } \\
\text { relacionadas con el suicidio }\end{array}$ & Intención de morir \\
\hline \multirow{9}{*}{$\begin{array}{l}\text { Conductas } \\
\text { relacionadas } \\
\text { con el suicidio }\end{array}$} & \multirow{3}{*}{ Autolesión } & Autolesión tipo I (sin lesiones) & \multirow{3}{*}{ Sin intención suicida } \\
\hline & & Autolesión tipo II (con lesiones) & \\
\hline & & $\begin{array}{l}\text { Muerte autoinfligida involuntaria } \\
\text { (con lesiones fatales) }\end{array}$ & \\
\hline & \multirow{3}{*}{$\begin{array}{l}\text { Conducta indeterminada } \\
\text { relacionada con el suicidio }\end{array}$} & $\begin{array}{l}\text { Conducta relacionada con el suicidio } \\
\text { indeterminada tipo I (sin lesiones) }\end{array}$ & \multirow{3}{*}{$\begin{array}{l}\text { Con intención suicida } \\
\text { indeterminada }\end{array}$} \\
\hline & & $\begin{array}{l}\text { Conducta relacionada con el suicidio } \\
\text { indeterminada tipo II (con lesiones) }\end{array}$ & \\
\hline & & $\begin{array}{l}\text { Muerte autoinfligida con intención } \\
\text { indeterminada (con lesiones fatales) }\end{array}$ & \\
\hline & \multirow{3}{*}{ Intento suicida } & Intento de suicidio tipo I ( $\sin$ lesiones) & \multirow{3}{*}{ Con intención suicida } \\
\hline & & Intento de suicido tipo II (con lesiones) & \\
\hline & & Suicidio (con lesiones fatales) & \\
\hline
\end{tabular}

En esa propuesta hubo más precisión respecto a la ideación suicida; sin embargo, la categoría de comunicaciones asociadas al suicidio podría generar confusión, pues agrega conductas y cogniciones. Además, la nomenclatura utiliza al menos un término que se considera inaceptable por otros autores, sobre lo que se profundizará en el apartado correspondiente (Crosby et al., 2011; Posner et al., 2014).

La nomenclatura de Silverman et al. (2007a, 2007b) es una de las que cuenta con la mayor aceptación internacional, lo que ha llevado a los expertos en el área a medir su aplicabilidad. Un ejemplo de ello son los resultados reportados por Kattimani, Bharadwaj, Sarkar y Mukherjee (2015), quienes midieron la confiabilidad entre observadores al utilizar dicha nomenclatura, hallando que las categorías de ideas y conductas asociadas al suicidio tienen un nivel de concordancia moderado, mientras que las comunicaciones relacionadas con el mismo mostraron un nivel de concordancia bajo.

\section{La clasificación de 2011}

El Sistema de Clasificación de Violencia Autodirigida (SCVA) surgió como producto de una iniciativa del Centro de Control y Prevención de Enfermedades en Estados Unidos para crear una nomenclatura que fuera más aceptada. Esta herramienta, que cuenta con la aprobación de cuatro agencias federales del gobierno de ese país, está fundamentada en la definición de violencia de la OMS ( $c f$. Matarazzo, Clemans, Silverman y Brenner, 2013).

Es pertinente aclarar que la Organización definió la violencia como el uso intencional de la fuerza física o las amenazas contra uno mismo $\mathrm{u}$ otra persona, grupo o comunidad que tiene como consecuencia, o es muy probable que tenga, traumatismos, daños psicológicos, problemas de desarrollo o la muerte (Krug, Dahlberg, Mercy, Zwi y Lozano, 2002), definición que justifica el nombre de la clasificación.

En el SCVA, las conductas asociadas al suicidio son las siguientes: la violencia autodirigida, que se entiende como un comportamiento que deliberadamente causa lesiones o genera la posibilidad de lesionarse a sí mismo; tal violencia puede ser suicida (si existe evidencia implícita o explícita de intención suicida), no suicida (si no existe evidencia implícita o explícita de intención suicida) o indeterminada (si no se puede decretar la intención suicida con la evidencia disponible), y la conducta preparatoria, que involucra cualquier acción que acondiciona el escenario para participar en la violencia autodirigida, esto es, antes de ocurrir las posibles lesiones por ejemplo, comprar un arma, en la que se articula un método o la redacción de una nota póstuma en que la persona advierte que se prepara a morir (Crosby et al., 2011). 
La ideación suicida involucra los pensamientos acerca de participar en cualquier conducta relacionada con el suicidio. Así, la ideación violenta autodirigida no suicida se refiere a los pensamientos relativos al deseo de participar en comportamientos autoinfligidos potencialmente dañinos, pero sin evidencia de intención suicida.
La intención suicida es la evidencia pasada o presente, implícita o explícita, acerca de que el individuo desea morir y comprende las posibles consecuencias de sus acciones o posibles acciones, es decir, suicidarse. En la Tabla 3 se muestra el sistema de clasificación de violencia autodirigida.

Tabla 3. Sistema de clasificación de violencia autodirigida (Crosby et al., 2011).

\begin{tabular}{|c|c|c|}
\hline Tipo & Subtipo & Términos \\
\hline \multirow[b]{2}{*}{ Pensamientos } & Ideación violenta autodirigida no suicida & •Ideación violenta autodirigida no suicida \\
\hline & Ideación suicida & $\begin{array}{l}\text { - Ideación suicida sin intención suicida. } \\
\text { - Ideación suicida con intención suicida indeterminada. } \\
\text { - Ideación suicida con intención suicida. }\end{array}$ \\
\hline \multirow{4}{*}{ Conductas } & Preparatoria & $\begin{array}{l}\text { - Violencia autodirigida no suicida, preparatoria. } \\
\text { - Violencia autodirigida indeterminada, preparatoria. } \\
\text { - Violencia autodirigida suicida, preparatoria. }\end{array}$ \\
\hline & $\begin{array}{l}\text { Violencia autodirigida no suicida } \\
\text { (Sin intención suicida) }\end{array}$ & $\begin{array}{l}\text { - Violencia autodirigida no suicida sin lesión. } \\
\text { - Violencia autodirigida no suicida sin lesión interrumpida } \\
\text { por sí mismo u otros. } \\
\text { - Violencia autodirigida no suicida con lesión. } \\
\text { - Violencia autodirigida no suicida con lesión interrumpida } \\
\text { por sí mismo u otros. } \\
\text { - Violencia autodirigida no suicida fatal. }\end{array}$ \\
\hline & $\begin{array}{l}\text { Violencia autodirigida indeterminada } \\
\text { (Intención suicida indeterminada) }\end{array}$ & $\begin{array}{l}\text { - Violencia autodirigida indeterminada sin lesión. } \\
\text { - Violencia autodirigida indeterminada sin lesión interrumpida } \\
\text { por sí mismo u otros. } \\
\text { - Violencia autodirigida indeterminada con lesión. } \\
\text { - Violencia autodirigida indeterminada con lesión interrumpida } \\
\text { por sí mismo u otros. } \\
\text { - Violencia autodirigida indeterminada fatal. }\end{array}$ \\
\hline & $\begin{array}{l}\text { Violencia autodirigida suicida } \\
\text { (Con intención suicida) }\end{array}$ & $\begin{array}{l}\text { - Intento de suicidio sin lesión. } \\
\text { - Intento de suicidio sin lesión interrumpido por sí mismo } \\
\text { u otros. } \\
\text { - Intento de suicidio con lesión. } \\
\text { - Intento de suicidio con lesión interrumpido por otros. } \\
\text { - Suicidio. }\end{array}$ \\
\hline
\end{tabular}

Similar a las otras propuestas, los términos están regulados por la intención de suicidio y la presencia de lesiones; sin embargo, la interrupción del comportamiento es un modulador que se agregó después (Crosby et al., 2011). La principal limitante que se considera para esta clasificación es en cuanto al uso de la etiqueta "violencia autodirigida". Al analizar la definición de violencia de la OMS, parece comprensible y congruente ( $c f$. Krug et al., 2002). Sin embargo, esta definición de violencia excluye los diferentes tipos de violencia que la legislación mexicana ha enlistado: psicológica, patrimonial, económica y sexual $(\mathrm{H}$. Congreso de la Unión, 2018).

En el mismo sentido, es oportuno revisar una definición de violencia que incluya los mencio- 
nados tipos de violencia y que cuente con mayor aceptación. De esta manera, se considerará violencia cualquier comportamiento que tiene como objetivo ejercer poder mediante el empleo de la fuerza física, psíquica, económica o política.

La violencia requiere de dos o más posiciones en los que existe un desbalance de poder que coloca a una como superior y a la otra como inferior (Ruiz y Blanco, 2004). Considerando esta definición, en los pensamientos y conductas relacionados con el suicidio no existe un desbalance de poder entre dos partes; además, el objetivo de cualquier conducta o pensamiento relacionada con el suicidio difícilmente será que un individuo ejerza poder sobre sí mismo.

Para cerrar este apartado es oportuno describir que a pesar de la limitante antes descrita, el sistema de clasificación de violencia autodirigida es una propuesta con un elevado nivel de aceptación e implementación a nivel internacional. No obstante, a opinión de los autores del presente texto, dicha limitante involucra un error conceptual (Barczyk, Piper, Duzinski, Klingensmith y Lawson, 2018; Brenner et al., 2011).

\section{Términos inaceptables}

Con base en la lectura de lo escrito hasta este punto, el lector podrá inferir la mayor parte de los términos que la literatura y los expertos en el campo han sugerido descartar. Sin embargo, se decidió abordar este apartado debido a su importancia.

Ya Silverman (2006) ha destacado la relevancia de disminuir la cantidad de sinónimos que se han utilizado para referirse a los pensamientos y conductas relacionadas con el suicidio y el acto en sí. Además, también hay consenso respecto a que ciertos términos son inadecuados, como los que se enlistan a continuación.

Suicidio consumado. Con base en las definiciones de suicidio revisadas, se ha aclarado que se trata de una muerte autoinfligida; por ende, la frase "suicidio consumado" es un pleonasmo. Además, tal frase hace referencia a lograr un objetivo, pero el resultado no fue el deseado en aquellas personas cuya meta era diferente a quitarse la vida. Se sugiere, por consiguiente, emplear el termino suicidio (Crosby et al., 2011; Posner et al., 2014).

Instituto de Investigaciones Psicológicas - Universidad Veracruzana
Conducta parasuicida, gesto suicida y amenaza suicida. Estos términos atribuyen una connotación peyorativa a la persona e invalidan, minimizan o trivializan el malestar que aquella experimenta, pues se califica su comportamiento como un acto de manipulación. La OMS (2012) ha sugerido sustituir "conducta parasuicida" por autolesiones, según la nomenclatura de 2007 (Silverman et al., 2007a) o violencia autodirigida no suicida, con base en la clasificación de 2011 (Crosby et al., 2011; Posner et al., 2014).

$\mathrm{Al}$ respecto del término "gesto suicida", a partir de la nomenclatura de Silverman et al. (2007a) se descartó su uso, de manera que se sugiere sustituirlo por autolesión o conducta violenta autoinfligida. Y en cuanto a "amenaza suicida", en la clasificación de Crosby et al. (2011) se incorporó como un término inaceptable por la connotación peyorativa que implica, sugiriendo sustituirlo por violencia autodirigida suicida o violencia autodirigida no suicida. No obstante, aún quedan ciertos comportamientos que podrían englobarse en conducta preparatoria en tanto que no hay una categoría específica al respecto. Sin embargo, este último punto se aborda en la discusión. El término "intento de suicidio fallido" da una impresión negativa sobre el desempeño de la persona al ejecutar una conducta al implicar un esfuerzo fracasado en la obtención de una meta; por consiguiente, se sugiere sustituirlo por intento de suicidio con o sin lesiones (Crosby et al., 2011).

\section{DISCUSIÓN}

Como se describió al principio, la definición de suicidio parece haber alcanzado un consenso internacional entre los diferentes grupos de investigación en el área. En efecto, utilizar cualquiera de las definiciones enlistadas conducen a identificar el mismo componente: la muerte de la persona, este resultado fue autoinfligido y esa persona tenía la intención de quitarse la vida. La sugerencia de los autores es utilizar cualquier definición que incorpore al menos estos componentes.

En lo que respecta a la nomenclatura de O'Carroll et al. (1996), se sugiere no utilizarla debido las limitaciones descritas; de igual modo, al 
compararla con las dos propuestas restantes es evidente que se torna insuficiente. Sin embargo, se destaca su relevancia como un estudio pionero en el campo, el cual se ha trasformado en una referencia esencial para los profesionales de la salud interesados. La nomenclatura de 1996, en opinión de los presentes autores, es la piedra angular sobre la que se han cimentado las diversas propuestas.

Otro punto en el que coinciden los diferentes grupos de investigación es la terminología que se propone no utilizar, por ejemplo: suicido consumado, conducta parasuicida y gesto suicida. Respecto a la amenaza suicida aún hay discrepancias, especialmente entre la nomenclatura de Silverman et al. (2007a, 2007b) y la clasificación de Crosby et al. (2011). Para este tema particular, los presentes autores están en favor de descartar el término "amenaza suicida".

Una de las conclusiones principales de este trabajo es sugerir a los profesionales de la salud en México inclinarse por la propuesta de Silver- man et al. (2007a, 2007b) o por la de Crosby et al. (2011). Aun así, también se sugiere que el uso de cualesquiera de estas propuestas se haga a la luz de las limitantes ya descritas, además de considerar los siguientes puntos.

Ambas propuestas coinciden en tres categorías para los comportamientos relacionados con el suicidio, una de las cuales incorpora la intencionalidad suicida, otra en la que no hay la intención de quitarse la vida, y una tercera según la cual no es posible determinar la presencia o ausencia de la intención suicida. En estas tres categorías, también concuerdan los autores en que existen comportamientos mediante los cuales se causan lesiones, otros en los que no las hay, y finalmente conductas que tienen un desenlace fatal: la muerte del individuo. La única diferencia que se identifica es meramente de etiquetación, a excepción del intento suicida y el suicidio, lo que se muestra en la Tabla 4.

Tabla 4. Comparación de conductas relacionadas con el suicidio.

\begin{tabular}{|c|c|c|}
\hline Conductas & Silverman et al. (2007a, 2007b) & Crosby et al. (2011) \\
\hline Sin intención suicida & Autolesiones & Violencia autodirigida no suicida \\
\hline Sin lesiones & Autolesión Tipo I & $\begin{array}{l}\text { Violencia autodirigida no suicida } \\
\text { sin lesión }\end{array}$ \\
\hline Con lesiones & Autolesión Tipo II & $\begin{array}{l}\text { Violencia autodirigida no suicida } \\
\text { con lesión }\end{array}$ \\
\hline Con resultado fatal & Muerte autoinfligida involuntaria & $\begin{array}{l}\text { Violencia autodirigida no suicida } \\
\text { fatal }\end{array}$ \\
\hline Con intención indeterminada & $\begin{array}{l}\text { Conducta relacionada con el suicidio } \\
\text { indeterminada }\end{array}$ & Violencia autodirigida indeterminada \\
\hline Sin lesiones & $\begin{array}{l}\text { Conducta relacionada con el suicidio } \\
\text { indeterminada Tipo I }\end{array}$ & $\begin{array}{l}\text { Violencia autodirigida indeterminada } \\
\text { sin lesión }\end{array}$ \\
\hline Con lesiones & $\begin{array}{l}\text { Conducta relacionada con el suicidio } \\
\text { indeterminada Tipo II }\end{array}$ & $\begin{array}{l}\text { Violencia autodirigida indeterminada } \\
\text { con lesión }\end{array}$ \\
\hline Con resultado fatal & $\begin{array}{l}\text { Muerte autoinfligida con intención } \\
\text { indeterminada }\end{array}$ & $\begin{array}{l}\text { Violencia autodirigida indeterminada } \\
\text { fatal }\end{array}$ \\
\hline Con intención suicida & Intento de suicidio & Violencia autodirigida suicida \\
\hline Sin lesiones & Intento de suicidio Tipo I & Intento de suicidio sin lesión \\
\hline Con lesiones & Intento de suicidio Tipo II & Intento de suicidio con lesión \\
\hline Con resultado fatal & Suicidio & Suicidio \\
\hline
\end{tabular}

Donde hubo discrepancias fue en las conductas asociadas al suicidio que no implican la posibilidad de una lesión en el evento. Crosby et al. (2011) propusieron la categoría de conducta "pre- paratoria", con la que los presentes autores concuerdan. Silverman et al. (2007a, 2007b) incluyeron la categoría de "comunicaciones suicidas", según la cual la amenaza suicida ${ }^{2}$ no coincide con

\footnotetext{
${ }^{2}$ Es indispensable recordar que este es un término que se sugiere descartar.
} 
la conducta preparatoria. Además, en la categoría de comunicaciones suicidas se incorpora el plan suicida, que se refiere a un evento cognitivo.

La propuesta de los autores, que no va más allá de una mera opinión, es conservar el apartado de conducta preparatoria, además de incorporar las conductas a las que se refiere la amenaza suicida como un comportamiento relacionado con el suicidio, pero con el término "comunicación suicida", o tal vez un concepto meramente conductal como el de "conducta verbal" (Skinner, 1957) relacionada con el suicidio, el cual podría incorporar el modulador de la intención suicida.

En referencia a los pensamientos asociados con el suicidio, ambas propuestas coinciden en que puede haber ideación suicida con intención, sin intención o con intención indeterminada. Los presentes autores concuerdan en que es oportuno conservar el término "ideación violenta autodirigida no suicida”. Aun así, consideran que es pertinente cambiar la etiqueta; además, aparece en la literatura la planeación suicida como una variable de interés, con lo que están de acuerdo.

Como comentario final, es oportuno aclarar que son múltiples las disciplinas que estudian el fenómeno analizado (psicología, sociología, derecho, medicina, por solo mencionar algunas), por lo que hay muy diferentes perspectivas. Sin embargo, no fue objetivo del presente escrito escudriñar entre los diferentes campos de estudio. Si bien este texto se elaboró teniendo como base la psicología de la salud, se espera que pueda ser de utilidad para los diferentes profesionales de la salud.

\section{AGRADECIMIENTO}

Se agradece al Consejo Nacional de Ciencia y Tecnología haber brindado la beca para estudios de doctorado del primer autor (Núm. de Becario: 280353), a través del apoyo Núm. 414023.

Citación: Rangel V., J.N. y Jurado C., S. (2022). Definición de suicidio y de los pensamientos y conductas relacionadas con el mismo: una revisión. Psicología y Salud, 32(1), 39-48. https://doi.org/10.25009/pys.v32i1.2709.

\section{REFERENCIAS}

Barczyk, A.N., Piper, K., Duzinski, S.V., Klingensmith, M. y Lawson, K.A. (2018). Youth suicide attempt nomenclature used in two central Texas hospitals. Crisis, 39. 461-468.

Borges, G., Orozco, R., Benjet, C. y Medina-Mora, M.E. (2012). Suicidio y conductas suicidas en México: retrospectiva y situación actual. Salud Publica de México, 52(4), 292-304.

Brenner, L.A., Breshears, R.E., Betthauser, L.M., Bellon, K.K., Holman, E., Harwood, J.E., Silverman, M.M., Huggins, J. y Nagamoto, H.T. (2011). Implementation of a suicide nomenclature within two VA healthcare settings. Journal of Clinical Psychology in Medical Settings, 18, 116-128.

Crosby, A.E., Ortega, L. y Melanson, C. (2011). Self-directed violence surveillance: Uniform definitions and recommended data elements (Version 1.0). Atlanta (GA): Centers for Disease Control and Prevention, and National Center for Injury Prevention and Control.

De Leo, D., Burgis, S., Bertolote, J., Kerkhof, A.D.M. y Bille-Brahe, U. (2004). Definitions of suicidal behavior. En D. de Leo, U. Bille-Brahe, A. D M. Kerkhof y A. Schmidtke (Eds.): Suicidal behavior: Theories and research findings (pp. 17-39). Washington, D.C: Hogrefe \& Huber.

Durkheim, E. (1897). Le suicide. Étude de sociologie. Paris: Félix Alcan.

Glasziou, P., Del Mar, C. y Salisbury, J. (2003). Evidence-based medicine workbook. Finding and applying the best evidence to improve patient care. London: BMJ Books.

H. Congreso de la Unión (2018). Ley general de acceso de las mujeres a una vida libre de violencia. Diario Oficial de la Federación (13 de abril). Ciudad de México. 
Instituto Nacional de Estadística e Informática (2019, 12 de febrero). Datos. Mortalidad. Tabulados. Mortalidad general. Ciudad de México: INEGI. Recuperado de https://www.inegi.org.mx/app/tabulados/pxweb/inicio.html?rxid=91a63b16-b544-4b50 -8b37-90d5868e9620ydb=Saludypx=Mental_06.

Kattimani, S., Bharadwaj, B., Sarkar, S. y Mukherjee, A. (2015). Interrater reliability of the Silverman et al. nomenclature for suicide-related ideations, behaviors, and communications. Crisis, 36(1), 61-64.

Kerlinger, F.N. y Lee, H.B. (2002). Investigación del comportamiento: Métodos de investigación en ciencias sociales. México: McGraw-Hill.

Krug, E.G., Dahlberg, L.L., Mercy, J.A., Zwi, A.B. y Lozano, R. (2002). World report on violence and health. Geneva: World Health Organization.

Matarazzo, B.B., Clemans, T.A., Silverman, M.M. y Brenner, L.A. (2013). The Self-Directed Violence Classification System and the Columbia Classification Algorithm for suicide assessment: A crosswalk. Suicide and Life-Threatening Behavior, 43(3), 235-249.

O’Carroll, P.W., Berman, A.L., Maris, R.W., Moscicki, E.K., Tanney, B.L. y Silverman, M.M. (1996). Beyond the tower of Babel: A nomenclature for suicidology. Suicide and Life-Threatening Behavior, 26(3), 237-252.

O’Connor, R.C. y Nock, M.K. (2014). The psychology of suicidal behavior. Lancet Psychiatry, 1, 73-85.

Organización Mundial de la Salud (OMS) (1990). Clasificación Estadística Internacional de Enfermedades y Problemas Relacionados con la Salud (10a revisión: CIE-10). Ginebra: OMS.

Organización Mundial de la Salud (2018b). CIE-11 para estadísticas de mortalidad y morbilidad. Ginebra: OMS. Recuperado de: https://icd.who.int/browse11/l-m/es psychosocial disorders. Suicide. Ginebra: OMS.

Posner, K., Brodsky, B., Yershova, K., Buchanan, J. y Mann, J. (2014). The classification of suicidal behavior. En M.K. Nock (Ed.): The Oxford handbook of suicide and self-injury (pp. 7-22). New York: Oxford University Press.

Rubin, A. y Bellamy, J. (2012). Practitioner's guide to using research for evidence-based practice. New York: Wiley \& Sons.

Ruiz J., C. y Blanco, P. (2004). La violencia contra las mujeres: prevención y detección. Cómo promover desde los servicios sanitarios relaciones autónomas, solidarias y gozosas. Madrid: Díaz de Santos.

Shneidman, E.S. (1985). Definition of suicide. New York: John Wiley \& Sons.

Silverman, M.M. (2006). The language of suicidology. Suicide and Life-Threatening Behavior, 36(5), 519-532.

Silverman, M.M., Berman, A.L., Sanddal, N.D., O'Carroll, P.W. y Joiner, T.E. (2007a). Rebuilding the tower of Babel: A revised nomenclature for the study of suicide and suicidal behaviors, Part 1: Background, rationale, and methodology. Suicide and Life-Threatening Behavior, 37(3), 248-263.

Silverman, M.M., Berman, B.L., Sanddal, N.D., O’Carroll, P.W. y Joiner, T.E. (2007b). Rebuilding the tower of Babel: A revised nomenclature for the study of suicide and suicidal behaviors, Part 2: Suicide-related ideations, communications, and behaviors. Suicide and Life-Threatening Behavior, 37(3), 264-277.

Silverman, M.M. y De Leo, D. (2016). Why there is a need for an international nomenclature and classification system for suicide. Crisis, 37(2), 83-87.

Skinner, B.F. (1957). Verbal behavior. New York: Appleton-Century-Crofts.

World Health Organization (2012). Public health action for the prevention of suicide. A framework. Geneva: WHO.

World Health Organization (2018a). Global health observatory data repository. Suicide rates, age-standardized. Data by country. Recuperado de http://apps.who.int/gho/data/node.main.MHSUICIDEASDR?lang=en.

World Health Organization and Panamerican Health Organization (1998). Primary prevention of mental, neurological and psycho-social disorders. Washington: WHO-PHO. 